\title{
Hard-wired Central Pattern Generator Hardware Network for Quadrupedal Locomotion Based on Neuron and Synapse Models
}

\author{
Akihiro Maruyama, , \# Tomoyasu Ichimura, ${ }^{* *}$ Yoshinobu MaEdA
}

\begin{abstract}
Many different quadrupedal walking patterns (gaits), such as "walking", "trotting", "bounding" and "galloping" can be generated by systems of coupled central pattern generators (CPG). However, the physiological mechanisms for the walking patterns are unclear. As a result, from an engineering viewpoint, many different mathematical models have been proposed to describe these walking patterns. In this report, we propose a hard-wired CPG network based on Rybak's model that can reproduce quadrupedal locomotion walking patterns. In this network, we use the beating model proposed by Hoshimiya et al. and the bursting model proposed by Maeda and Makino. The main purpose of this study was to reproduce the typical walking patterns; "walking" and "bounding", with a hardware model, and to switch between these patterns using only one parameter, which can be interpreted as voltage stimulation from the midbrain locomotor region. We found the transition from the "walking" to the "bounding" behavior to be due to a relative weakening of the coupling between the CPGs in the network by stimulation from the midbrain locomotor region.
\end{abstract}

Keywords: central pattern generator, walking gait, bounding gait, bursting neuron, negative resistance.

Adv Biomed Eng. 4: pp. 48-54, 2015.

\section{Introduction}

The walking pattern (or gait) of an animal, which depends on velocity, is primarily controlled by a coupled [1-10] or decoupled system [11] of central pattern generators (CPG). Many researchers have studied gait from an engineering viewpoint, because the physiological mechanisms and anatomical features are as yet unclear. Rybak et al. [8, 9] proposed a two-level CPG model based on the coupled Hodgkin-Huxley equation [12] and a half-center concept to explain the experimental results for the locomotion activity of decerebrate cats, i.e., whether or not spontaneous omission (or deletion) of the activity of a motor neuron causes phase reset of muscle contraction rhythms. Maeda [10] proposed an analog circuit system for Rybak's mathematical model [8, 9], using four kinds of model for beating and bursting neurons [13, 14] and synapse excitation and inhibition [10]. These are hardware models that use one CPG to drive the alternative activations of agonist and antagonist motor neurons.

In studying the diverse four-legged animal gaits [2] such as walking, trotting, pacing, cantering, galloping and bounding, it is necessary to have complex coupling between four CPGs that have the same neural network structure. The coupling of four CPGs to generate a specific animal gait may differ from that needed to generate other gaits. For example, coupling to generate walking

This study was presented at the Symposium on Biomedical Engineering 2014, Tokyo, September, 2014.

Received on July 31, 2014; revised on October 16, 2014; accepted on December 11, 2014.

* Graduate School of Science and Technology, Niigata University, Niigata, Japan.

** Oyama National College of Technology, Oyama, Japan.

\#2-8050, Ikarashi, Nishi-ku, Niigata 950-2181, Japan.

E-mail: f14c114a@mail.cc.niigata-u.ac.jp gait is structurally different from that to generate trotting or bounding gaits. Thus, one gait corresponds to one CPG network. However, this concept is physiologically unrealistic because the animal has to possess a large number of CPG networks corresponding to the number of gaits. In addition, if some of them are not used during the lifetime of the animal they would be superfluous. Therefore, a single hard-wired network that generates and switches between multiple animal gaits by changing a controllable parameter, such as an input stimulation, from the midbrain locomotor region to the $\mathrm{CPG}$ is required.

Collins and Richmond [3] suggested that switching between multiple animal gaits such as walking, trotting and bounding can be accomplished by changing the values of parameters in relatively simple mathematical models of the CPG. These models are coupled as a hard-wired network, but four or five parameters have to be changed simultaneously to switch between gaits. However, this is unrealistic from a physiological viewpoint because stability of the gait depends on the change being simultaneous, and there will be cases where this simultaneity is affected by noise. From an engineering viewpoint, a hard-wired CPG network, such as that presented by Collins and Richmond [3], is needed. However, switching gaits by changing a single parameter rather than a number of parameters is much more preferable.

In this paper, we are not concerned with the activity of a multi-jointed engineered limb, but are only interested in the coordination of neuronal oscillation patterns generated by CPG hardware models $[13,14]$. The main purpose of this study was to develop a hard-wired CPG hardware network for generating neuronal oscillation patterns corresponding to four-legged animal gaits, and to enable switching between two typical patterns corresponding to walking and bounding using only one parameter, the value of which is derived from voltage stimulation from the midbrain locomotor region. Hardware modeling has the following advantages: 1) the processing speed is faster than computer simulation, and 2) it can be easily applied to industrial technologies 


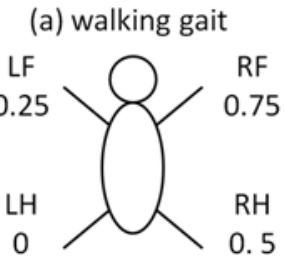

(b) bounding gait

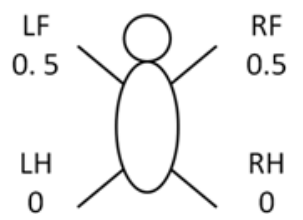

Fig. 1 Phase relations for quadrupedal gaits: (a) walking, and (b) bounding. The phases are normalized.

such as robotics [10]. Although the physiological CPG mechanisms, as mentioned above, are unclear, the proposed CPG hardware network facilitates the understanding of physiological phenomena, because unlike a software program, it qualitatively replicates the dynamics of the neuronal action potentials and synaptic potentials. Furthermore, we identified a relative weakening of the coupling in the CPG network in the transition from walking to bounding by stimulation from the midbrain locomotor region.

\section{Walking Patterns (Gaits)}

The quadrupedal walking and bounding patterns investigated in this paper are illustrated in Fig. 1. In the walking gait, each limb oscillates with phase shifts of 90 degrees between the left front (LF) limb and the left hind (LH) limb, the right hind (RH) limb and LF limb, the right front (RF) limb and RH limb, and the LH limb and RF limb. In the bounding gait, the right and left limbs are synchronized (in-phase) both for front and hind limbs, with the front and hind limbs, moving 180 degrees out of phase with each other (anti-phase). The walking gait is observed when the animal moves at a relatively slow speed. When the animal tries to go faster, it gallops using a similar gait known as the bounding gait. This is done to reduce energy consumption when in motion.

In this paper, we define the synchronized oscillation patterns generated by the hardware neuron models (described in the next section) as walking (for the oscillation pattern generating the walking gait) and bounding (for the oscillation pattern generating the bounding gait).

\section{Models}

\subsection{Hardware neuron models}

Figure 2a and $\mathbf{b}$ show the bursting neuron model proposed by Maeda and Makino [14] and the beating neuron model proposed by Hoshimiya et al. [13], respectively. The label $V_{m}$ represents the membrane potential at the inside of the membrane. The beating model (Fig. 2b) can generate action potentials repetitively by external current inputs. Basically this model consists of three parts. The first is a passive circuit comprising a resistor $R_{m}$ and a capacitor $C_{m}$ in parallel. The second is a nonlinear part for generating action potentials, which is a negative resistance circuit causing an inward current from the voltage $E$ when $V_{m}$ exceeds the threshold voltage (the base-emitter voltage of transistor $T r_{1}$ ). The third is a circuit that causes a delayed outward current (the collector-emitter current of transistor $T_{3}$ ) which functions as the refractoriness of the action potential. The bursting model (Fig. 2a) adds another delayed outward current that terminates the active phase of bursting and maintains the inactive (silent) phase until $V_{m}$ exceeds the threshold again. In order to operate these models, it is necessary to apply an external DC voltage, $V_{M L R}$, to the models across an (a)

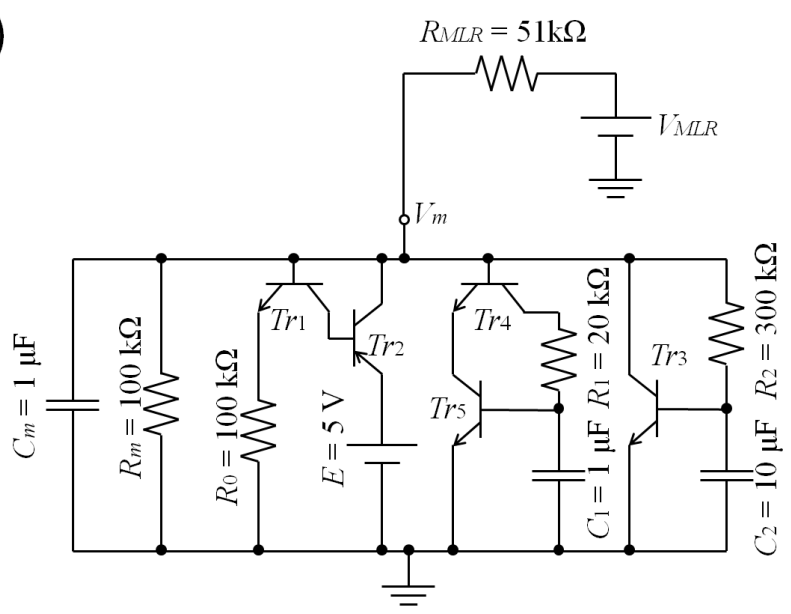

(b)

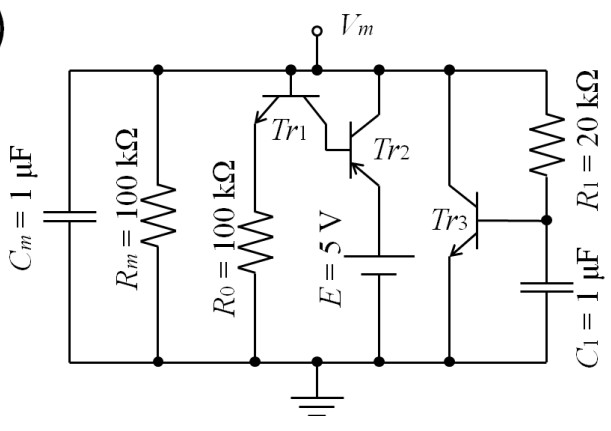

Fig. 2 (a) Bursting neuron model, and (b) beating neuron model. The top and bottom of the circuit represent the internal and external aspects, respectively, of the membrane. $V_{m}$ in volts represents the membrane potential. $E=5 \mathrm{~V}, C_{m}=C_{1}=1 \mu \mathrm{F}, C_{2}=10 \mu \mathrm{F}$, $R_{m}=R_{0}=100 \mathrm{k} \Omega, R_{1}=20 \mathrm{k} \Omega, R_{2}=300 \mathrm{k} \Omega, R_{M L R}=51 \mathrm{k} \Omega$.

input resistance of $51 \mathrm{k} \Omega$, which replicates an efferent stimulation from the midbrain locomotor region. In Fig. 2, the terminal, $V_{m}$, is connected to the synapse models described in the next section in which the hard-wired CPG hardware network is presented.

\subsection{Hardware synapse models}

The synapse excitation and inhibition hardware models proposed by Maeda [10] are shown in Fig. 3. The synapse excitation model excites the post-synaptic neuron model by passing current only when the pre-synaptic neuron model is firing. In contrast, the synapse inhibition model inhibits the post-synaptic neuron model by drawing current only when the pre-synaptic neuron model is firing. The input resistance, $R_{\text {in }}=10 \mathrm{M} \Omega$, is relatively large and little current flows in the models, so that the formation of action potentials in the pre-synaptic neuron model is not broken when transmitting information from the pre- to post-synaptic neuron models. Moreover, the current does not flow back from the post- to the pre-synaptic neuron model even if the post-synaptic neuron mod$\mathrm{el}$ is firing. The output resistance in the synapse excitation model had two values: $1 \mathrm{M} \Omega$ (weak) and $20 \mathrm{k} \Omega$ (strong), and that in the synapse inhibition model was $20 \mathrm{k} \Omega$. In the synapse inhibition model, we used photocouplers (TLP785) to stabilize the negative synaptic voltage, $E_{s y n}=-5 \mathrm{~V}$, which is regarded as being the reverse potential of the anion, whereas stabilized power supplies, $E_{s y n}=5 \mathrm{~V}$, can be used in the synapse excitation model. 
(a)

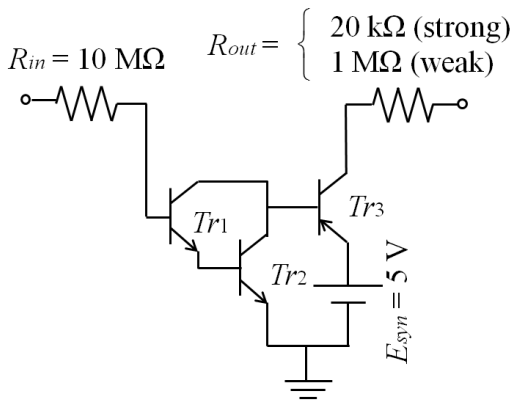

(b)

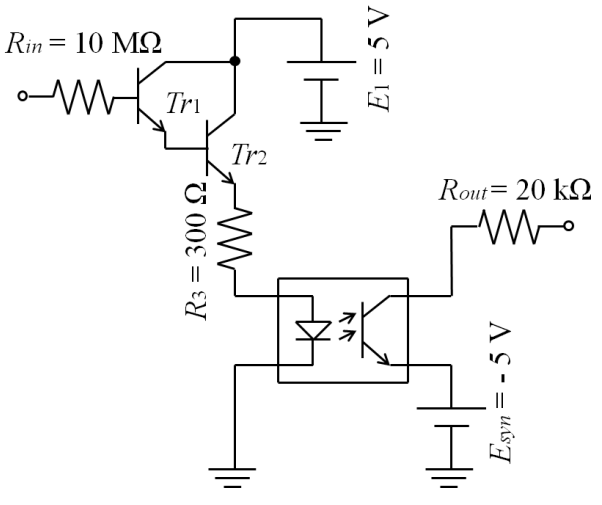

Fig. 3 (a) Synapse excitation model, and (b) synapse inhibition model. In the excitation model, $R_{\text {in }}=10 \mathrm{M} \Omega, R_{\text {out }}=20 \mathrm{k} \Omega$ (strong) or $1 \mathrm{M} \Omega$ (weak), $E_{s y n}=5 \mathrm{~V}$. In the inhibition model, $R_{\text {in }}=$ $10 \mathrm{M} \Omega, R_{\text {out }}=20 \mathrm{k} \Omega, R_{3}=300 \Omega, E_{1}=5 \mathrm{~V}, E_{\text {syn }}=-5 \mathrm{~V}$.

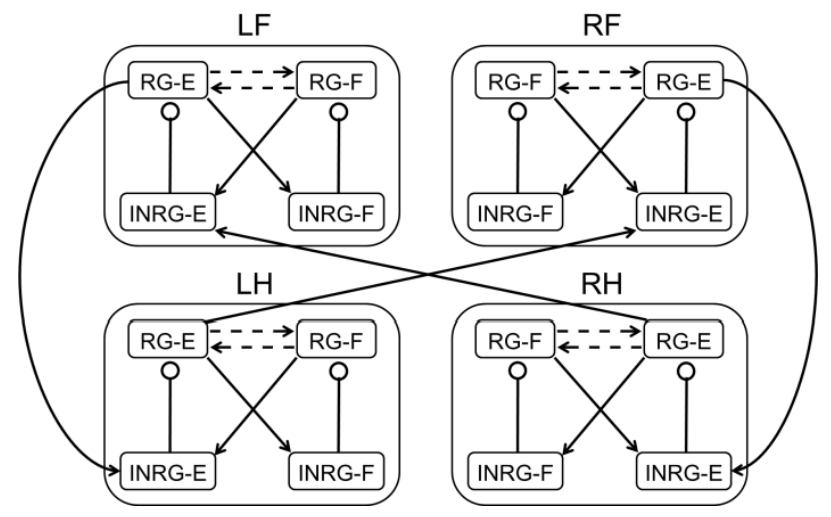

Fig. 4 Schematic diagram of the CPG network. The solid arrows, dashed arrows and balloon-lines represent strong excitation, weak excitation, and inhibition connections, respectively.

\subsection{Hard-wired CPG network}

Figure 4 shows a schematic diagram of the hard-wired CPG network that comprises four CPG sub-networks labeled LF, LH, RF and RH. We designed and constructed an actual circuit based on the schematic diagram shown in Fig. 4. In each CPG, there are two rhythm generators $(\mathrm{RG})$ with extensor (-E) and flexor (-F) sites that inhibit each other through interneuron models (INRG). This is regarded as a one-level CPG, reduced from the two-level CPG proposed by Rybak et al. [8, 9]. Inhibitory coupling means that the CPG generates alternative pulses between the agonist and antagonist motor neurons. The RGs and INRGs are simulated using the bursting neuron model (Fig. 2a) and the beating neuron

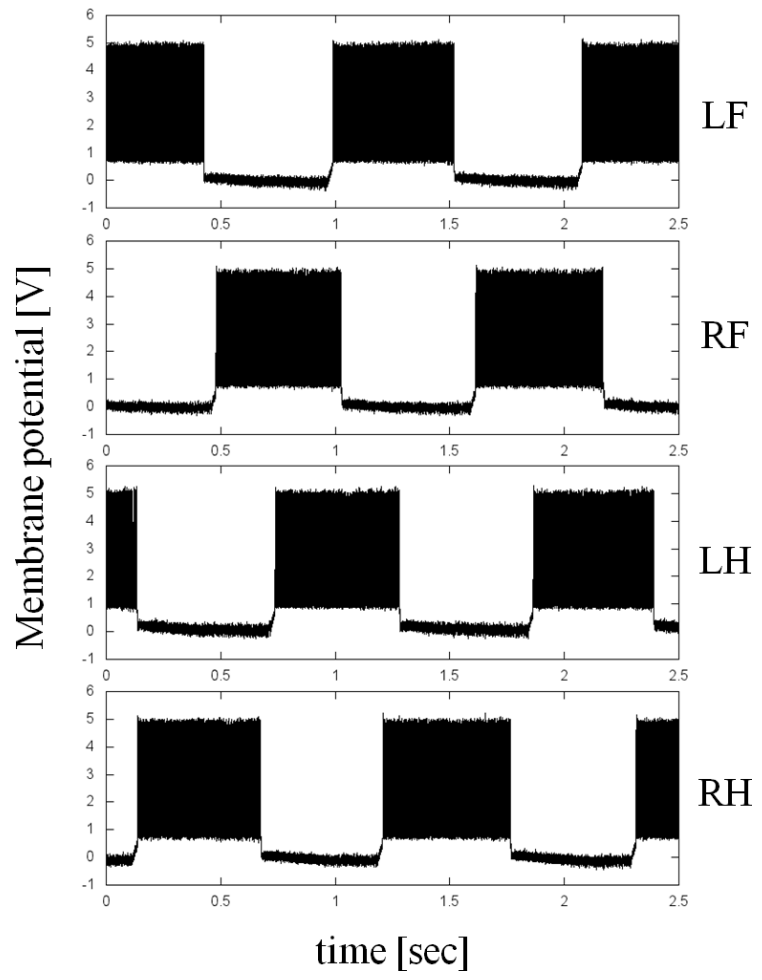

Fig. 5 Temporal waveforms for walking when the input DC voltage $V_{M L R}$ is $1.62 \mathrm{~V}$. From top to bottom, membrane potential $V_{m}$ at the RG-Es of "LF", "RF", "LH" and "RH".

model (Fig. 2b), respectively. In addition, the solid arrows, dashed arrows, and balloon-lines show strong excitation, weak excitation (Fig. 3a), and inhibition (Fig. 3b) connections, respectively, between the two hardware neuron models. Every CPG is unidirectionally coupled with another based on the network proposed by Collins and Richmond [3]; i.e., between LF, LH, RF, $\mathrm{RH}$ and LF in turn, using the strong synapse excitation model. In this paper, only the extensor sites were coupled. For example, RG-E in the left-front CPG (RG-E of LF) excites not only INRG-F in the same CPG but also INRG-E in the left-hind CPG (INRG-E of LH). Subsequently, INRG-E of LH inhibits RG-E in the same CPG (RG-E of LH).

In order to operate the CPG network, it is necessary to apply an external DC voltage, $V_{M L R}$, which is the unique control parameter in this study, to all the RGs across an input resistance of $51 \mathrm{k} \Omega$. This represents efferent stimulation from the midbrain locomotor region.

\section{Results}

The hard-wired CPG network (Fig. 4) produced a walking $\left(V_{M L R}=1.62 \mathrm{~V}\right)$ and a bounding $\left(V_{M L R}=3.52 \mathrm{~V}\right)$ behavior. The stationary temporal waveforms of the extensor sites (RG-Es) are shown in Fig. 5 for walking and Fig. 6 for bounding. For walking, periodic bursting discharges were observed in the LH, LF, RH, RF and LH legs in turn, with phase shifts of 90 degrees (Fig. 5). For bounding, the synchronized front limbs oscillate 180 degrees out of phase with the synchronized hind limbs (Fig. 6). On increasing $V_{M L R}$ quasi-statically from $1.62 \mathrm{~V}$ to $3.52 \mathrm{~V}$, the behavior changed from walking to bounding at around $2.8 \mathrm{~V}$ with a relatively short transient period. When $V_{M L R}$ was decreased back to 
$1.62 \mathrm{~V}$, bounding was maintained at $2.8 \mathrm{~V}$, and changed into walking only when $V_{M L R}<2.8 \mathrm{~V}$, after a relatively longer transient

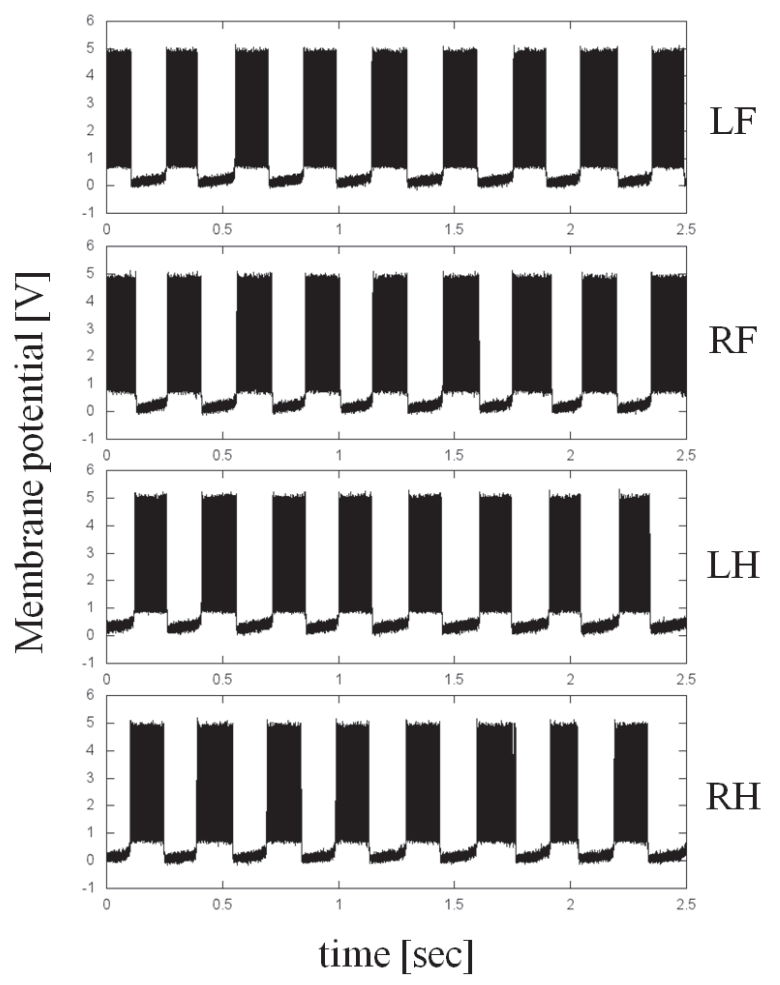

Fig. 6 Temporal waveforms for bounding when the input DC voltage $V_{M L R}$ is $3.52 \mathrm{~V}$. From top to bottom, membrane potential $V_{m}$ at the RG-Es of "LF", "RF", "LH" and "RH". period than that in the opposite direction. Thus, from these results, we learnt the following: first, the transient period is inversely proportional to $V_{M L R}$, and second, hysteresis is present in the transition between walking and bounding, with both walking and bounding being bi-stable in the dynamic system described by the hard-wired CPG network.

Figure 7 shows the walking-to-bounding transition when the input DC voltage $V_{M L R}$ was changed instantaneously from $1.62 \mathrm{~V}$ to $3.52 \mathrm{~V}$. In this case, we observed a rapid change from walking to bounding with a small transient period. The long transient period found when $V_{M L R}$ was changed quasi-statically was not found when $V_{M L R}$ was changed instantaneously.

\section{Discussion}

How does the hard-wired CPG network switch between walking and bounding by changing just one parameter, the input DC voltage $V_{M L R}$ ? In walking, all the limbs oscillate 90 degrees out of phase with each other. The limbs are not in-phase at any time. In bounding, however, the left limbs oscillate in-phase with the right limbs. On increasing $V_{M L R}$ from $1.62 \mathrm{~V}$ to $3.52 \mathrm{~V}$, a strong current is input into the RGs. Therefore, the bursting period, or the active phase period, becomes shorter and the number of firings in the active phase decreases [14]. This decrease in excitation at the RG level causes a decrease in excitation at the INRG level, so that the strength of the coupling between the RG levels becomes relatively weaker. We hypothesized that the weak coupling might allow in-phase synchronization between the left and right limbs, and as a result, walking can turn into bounding.

To verify our hypothesis, we remodeled one sub-network of the CPG network, as shown in Fig. 8. The bursting model (e.g., RG-E of LF) excites the beating model (e.g., INRG-E of LH)

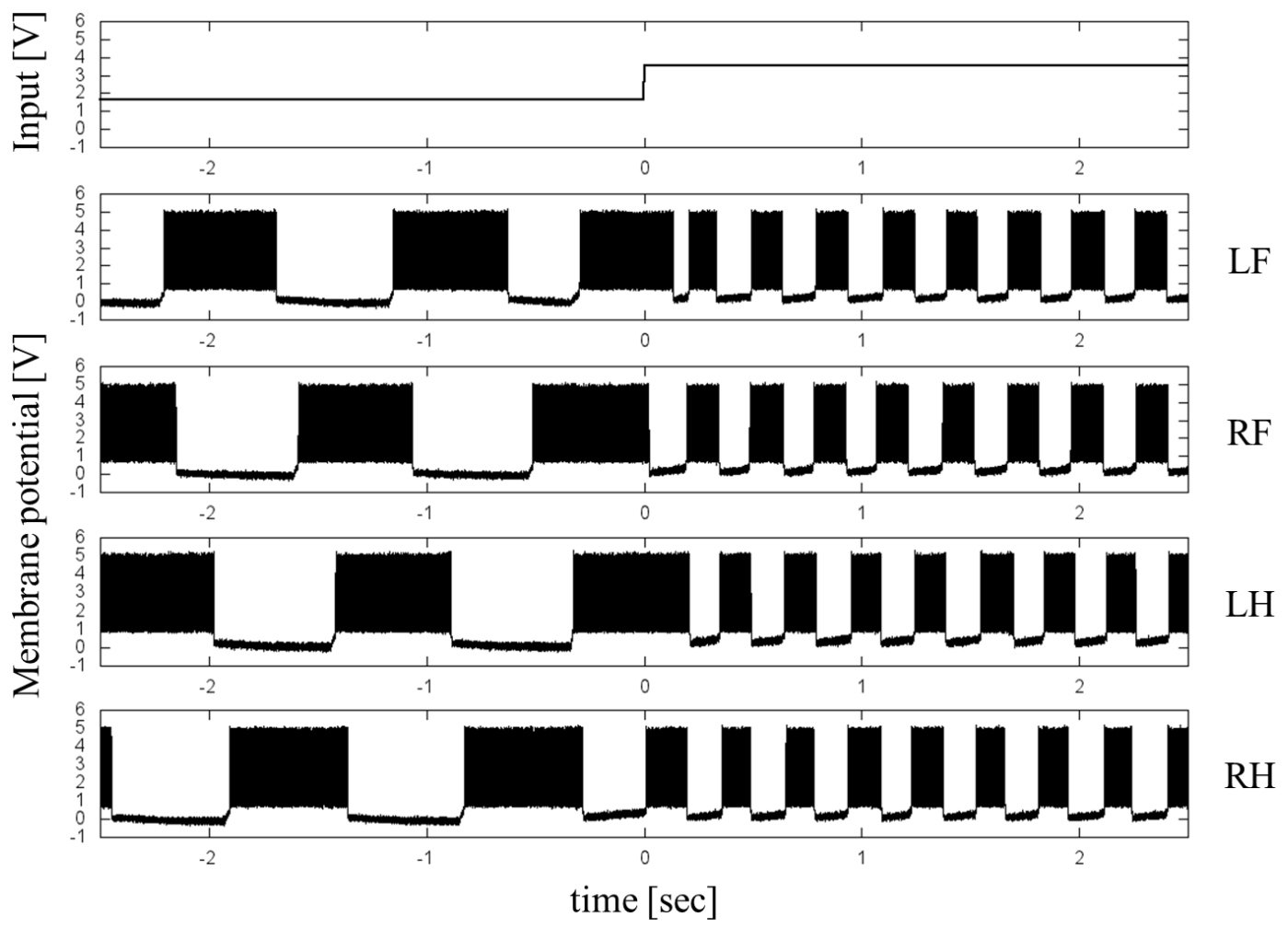

Fig. 7 Temporal waveforms for the walking-to-bounding transition when the input DC voltage $V_{M L R}$ is changed from $1.62 \mathrm{~V}$ to $3.52 \mathrm{~V}$. From top to bottom, input DC voltage $V_{M L R}$ and membrane potential $V_{m}$ at the RG-Es of "LF", "RF", "LH" and "RH". 


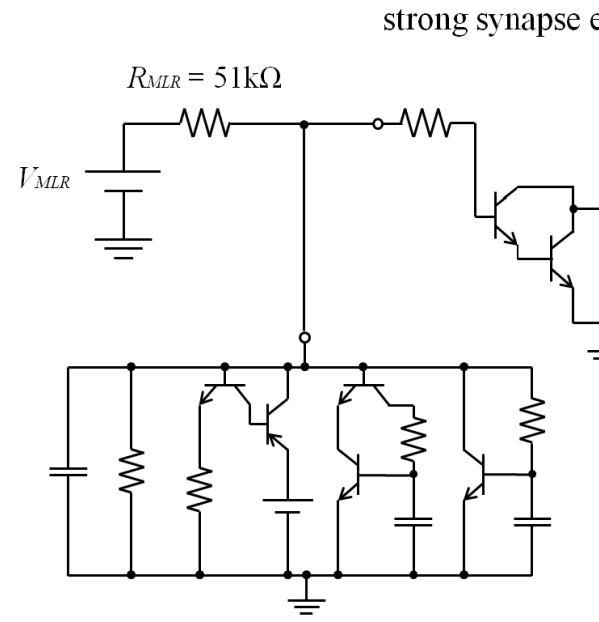

bursting neuron se excitation

\section{Engineering. Vol. 4, 2015.}




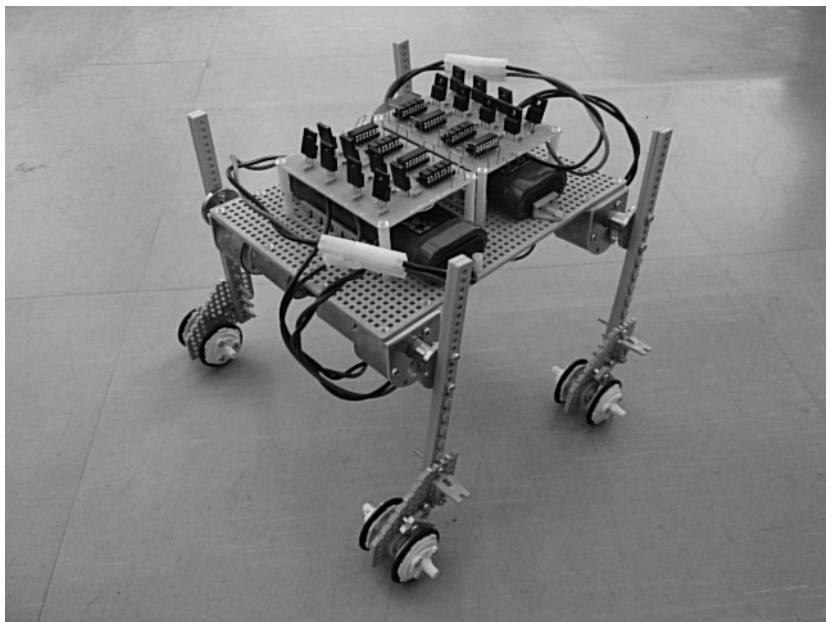

Fig. 11 Photograph of a quadrupedal robot. There are four DC motors, four motor drivers and LF, RF, LH and RH limbs.

In this study, we generated the electrical behavior or synchronized oscillation patterns corresponding to the walking and bounding gaits of four-legged animals using a hard-wired CPG hardware network. We are currently attempting to apply this hardwired CPG network to a quadrupedal walking robot, as shown in Fig. 11. One future development will be to reproduce actual voltage- controlled switchable gaits on the robot.

\section{Conclusion}

In this paper we propose a hard-wired CPG network based on neuronal firing, which can switch between the pattern for walking and the pattern for bounding by changing only one input parameter. We verified that, with the hard-wired CPG network generating the pattern corresponding to the walking gait, the pattern corresponding to the bounding gait appears if the strength of the CPG network is changed from being strongly coupled to being weakly coupled. In future work, we intend to make a robot switch between walking and bounding by varying the single parameter, $V_{M L R}$. We also plan to implement the CPG network in CMOS [15] in order to miniaturize the network and save energy.

\section{Conflict of Interest}

We have no conflicts of interest relationship with any companies or commercial organizations based on the definition of Japanese Society of Medical and Biological Engineering.

\section{References}

1. Taga G, Yamaguchi Y, Shimizu H: Self-organized control of bipedal locomotion by neural oscillators in unpredictable environment. Biol Cybern. 65, pp. 147-159, 1991.

2. Collins JJ, Stewart IN: Coupled nonlinear oscillators and the symmetries of animal gaits. J Nonlinear Sci. 3, pp. 349-392, 1993.

3. Collins JJ, Richmond SA: Hard-wired central pattern generators for quadrupedal locomotion. Boil Cybern. 71, pp. 375-385, 1994.

4. Golubitsky M, Stewart I, Buono P, Collins JJ: Symmetry in locomotor central pattern generators and animal gaits. Nature. 401, pp. 693-695, 1999.
5. Nishii J: Legged insects select the optimal locomotor pattern based on the energetic cost. Biol Cybern. 83, pp. 435-442, 2000.

6. Ivashko DG, Prilutsky BI, Markin SN, Chapin JK, Rybak IA: Modeling the spinal cord neural circuitry controlling cat hindlimb movement during locomotion. Neurocomputing. 52-54, pp. 621-629, 2003.

7. Nakada K, Asai T, Amemiya Y: Biologically- inspired locomotion controller for a quadruped walking robot: Analog IC implementation of a CPG-based controller. J Robotics Mechatronics. 16(4), pp. 397-403, 2004.

8. Rybak IA, Stecina K, Shevtsova NA, McCrea DA: Modelling spinal circuitry involved in locomotor pattern generation: insights from deletions during fictive locomotion. J Physiol. 577(2), pp. 617-639, 2006.

9. Rybak IA, Stecina K, Shevtsova NA, McCrea DA: Modelling spinal circuitry involved in locomotor pattern generation: insights from the effects of afferent stimulation. J Physiol. 577(2), pp. 641-658, 2006.

10. Maeda Y: A hardware neuronal network model of a two-level central pattern generator. Trans. Jpn Soc Med Biol Eng. 46(5), pp. 496-504, 2008.

11. Owaki D, Kano T, Nagasawa K, Tero A, Ishiguro A: Simple robot suggests physical interlimb communication is essential for quadruped walking. J R Soc Interface. 10, http://dx.doi.org/10.1098/ rsif.2012.0669, 2012.

12. Hodgkin AL, Huxley AF: A quantitative description of membrane current and its application to conduction and excitation in nerve. J Physiol. 117, pp. 504-544, 1952.

13. Hoshimiya N, Yoshida S, Shogen K, Matsuo T: Two-terminal electronic circuit neuron model with excitable membrane V-I- $t$ characteristics. Biol Cybern. 35, pp. 125-130, 1979.

14. Maeda Y, Makino H: A pulse-type hardware neuron model with beating, bursting excitation and plateau potential. BioSystems. 58, pp. 93-100, 2000.

15. Saeki K, Sekine Y: CMOS implementation of neuron models for an artificial auditory neural network. IEICE Trans Fundamentals. E86-A(2), pp. 424-427, 2003.

\section{Akihiro MaruYama \\ Received a B.E. (2014) in Biocybernetics from Ni- igata University. Presently, he is Graduate Student in Graduate School of Science and Technology, Niigata University. His research field is circuit de- sign of neural network.}

\section{Tomoyasu ICHIMURA}

Received a M.S. (1997) and a Ph.D. (2001) in Information Science and Engineering from Niigata University. From 2001 to 2002, he was a Postdoctoral Fellow with the Department of Mechanical Engineering, University of Illinois at Chicago. He is currently an Associate Professor with the De-

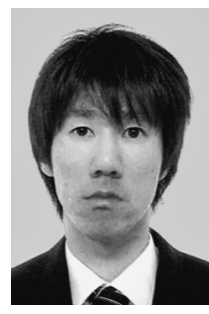
partment of Innovative Electrical and Electronic Engineering, Oyama National College of Technology. His research interests include mobile robots. He is a regular member of the Robotics Society of Japan (RSJ) and the Institute of Electronics, Information and Communication Engineers (IEICE). 


\section{Yoshinobu MaEda}

Received a M.S. (1995) and a Ph.D. (1998) in Biophysical Engineering from Osaka University. He was JSPS Research Fellow (1995-97) in Osaka University, Research Associate (1998-2005) in Niigata University, and Visiting Associate Professor (2007) in Hiroshima University. Presently, he is

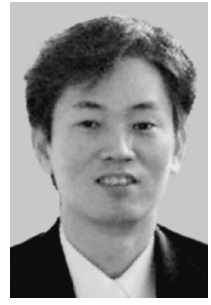

Associate Professor in Graduate School of Science and Technology, Niigata University. His research fields are neuro- and socio-dynamics, human interface and assistive technology. He is a regular member of IEICE, JSMBE, IPSJ, JSWSAT and IEE of Japan. 